\title{
The importance of audiometric monitoring in patients with multidrug-resistant tuberculosis
}

\author{
Karla Anacleto de Vasconcelos ${ }^{[1]}$, Silvana Maria Monte Coelho Frota ${ }^{[1],[2]}$, \\ Antonio Ruffino-Netto ${ }^{[3]}$ and Afrânio Lineu Kritski ${ }^{[2],[4]}$
}

\begin{abstract}
[1]. Departamento de Fonoaudiologia, Hospital Universitário Clementino Fraga Filho, Faculdade de Medicina, Universidade Federal do Rio de Janeiro, Rio de Janeiro, RJ, Brasil. [2]. Faculdade de Medicina, Universidade Federal do Rio de Janeiro, Rio de Janeiro, RJ, Brasil.

[3]. Faculdade de Medicina de Ribeirão Preto, Universidade de São Paulo, Ribeirão Preto, SP, Brasil.

[4]. Programa de Pós-Graduação em Clínica Médica, Faculdade de Medicina, Universidade Federal do Rio de Janeiro, Rio de Janeiro, RJ, Brasil
\end{abstract}

\begin{abstract}
Introduction: A total of 771 cases of multidrug-resistant tuberculosis (MDR-TB) were reported in Brazil in 2014. Treatment of MDR-TB with aminoglycosides can produce serious side effects such as permanent and irreversible hearing loss, which occurs in $5-64 \%$ of cases, and severely compromise patient quality of life. The goal of this research was to evaluate auditory and vestibular side effects in patients treated for MDR-TB and to identify associations between these complaints and the type of aminoglycoside used. Methods: We performed a retrospective review of 599 medical records from patients with MDR-TB who were treated at the Hélio Fraga/Fiocruz Reference Center between 2006 and 2010. Cases without auditory or vestibular complaints and patients who were not treated with aminoglycoside drugs were excluded from the study. Results: Of 164 eligible cases, 55 (33.5\%) reported an auditory or vestibular complaint and medication was subsequently suspended, although hearing damage was not confirmed in all cases. Audiometric testing confirmed hearing loss in $11(21.7 \%)$ of 12 cases submitted for evaluation. Hearing loss related to ototoxicity was confirmed in $15(62.5 \%)$ cases. Tinnitus was significantly associated with the use of amikacin and streptomycin. Conclusions: Evaluations of ototoxicity symptoms were not usually reported in the routine care of patients with MDR-TB. Complaints of tinnitus were associated with amikacin and streptomycin use. These results require confirmation in future studies.
\end{abstract}

Keywords: Aminoglycoside. Hearing. Hearing loss. Tuberculosis.

\section{INTRODUCTION}

In recent years, the increasing incidence of multidrugresistant tuberculosis (MDR-TB) has become a significant concern for policy makers. The World Health Organization ${ }^{1}$ estimated that in 2014, there were 480,000 new cases of MDRTB worldwide and approximately 190,000 deaths related to MDR-TB ${ }^{1}$. Brazil is ranked 17 among the 22 countries with the highest number of cases of MDR-TB, reporting 70,000 new cases and 4,600 related deaths annually ${ }^{1,2}$. Moreover, an increase in the number of MDR-TB cases was recently reported in Rio de Janeiro State in the year $2015^{2}$.

Patients with MDR-TB characterized by bacilli resistant to first-line treatments including rifampicin and isoniazid require the use of second-line medications such as aminoglycosides. Aminoglycosides have been used to treat TB for more than 40 years $^{3}$. These agents are cost-effective and widely used in low-

Corresponding author: Fga. Karla Anacleto de Vasconcelos.

e-mail: karla.fono@hotmail.com

Received 15 November 2016

Accepted 18 October 2017 and middle-income countries ${ }^{2,3}$. Yet, aminoglycoside use for the treatment of MDR-TB is associated with ototoxicity in 5-64\% in cases $^{3-11}$. Ototoxicity can produce hearing loss and associated psychosocial changes that negatively affect quality of life ${ }^{12}$.

Recent research has highlighted the importance of auditory monitoring in patients receiving aminoglycosides ${ }^{3,5,11}$. Auditory monitoring facilitates the early identification of injury before the onset of hearing loss of tonal thresholds in the range of conventional frequencies used in speech. Consequently, screening and appropriate treatment can reduce the effect of aminoglycoside-related ototoxicity on quality of life. There is little data on ototoxicity in patients receiving aminoglycosides for the treatment of MDR-TB in Brazil ${ }^{7,11}$. Therefore, the goal of this study was to retrospectively evaluate auditory and vestibular complaints in patients treated for MDR-TB at a reference center in Rio de Janeiro State, and to analyze relationships between these complaints and the type of aminoglycoside used.

\section{METHODS}

We retrospectively enrolled patients with MDR-TB who attended the outpatient clinic at Helio Fraga Reference Center/ FIOCRUZ between January 2006 and December 2010. Clinical 
and socio-demographic data were obtained from the medical records of the National Surveillance System for MDR-TB. Secondary data were obtained from clinic medical records. Clinical variables included hearing complaints such as hearing loss (difficulty hearing or understanding speech), tinnitus, and dizziness were verified in this study. Information about the discontinuation of aminoglycosides due to auditory or vestibular complaints and requests for audiometric evaluation were also recorded. Hearing test results were recorded from medical charts and analyzed separately for each ear. Cases of MDR-TB were excluded from the analysis if there were no auditory or vestibular data or if an aminoglycoside was not administered.

Associations between the most frequent symptoms and the variables studied (aminoglycoside, sex, age, schooling, previous use of an aminoglycoside, and previous treatment) were tested using Chi-square tests. The threshold for statistical significance was $p<0.05$. The epiinfo version 7 program was used.

\section{Ethical considerations}

This study was approved by the Research Ethics Committee of the Hospital Universitário Clementino Fraga Filho/Universidade Federal do Rio de Janeiro (approval no. 75676/12).

The procedures used were in accordance with the ethical standards of the responsible human experimentation committee (institutional, regional or national) and in accordance with the Declaration of Helsinki of 1964, revised in 1975, 1983, 1989, 1996 and 2000.

\section{RESULTS}

Of 599 medical records analyzed, 417 (69.6\%) were excluded from the analysis due to the absence of auditory/vestibular complaints and $10(1.7 \%)$ were excluded due to use of a nonaminoglycoside treatment regimen. In the remaining 172 (28.7\%) cases, amikacin was administered in $145(84.3 \%)$ patients and streptomycin was administered in 19 (11\%) patients (Figure 1).

One or more auditory/vestibular complaints were reported in 164 (95.3\%) cases; hypoacusis was reported in $116(70.7 \%)$ cases, tinnitus was reported in $88(53.6 \%)$ cases, and dizziness was reported in $28(17 \%)$ cases (Figure 2). Fifty-five (33.5\%) patients discontinued the medication after reporting a hearing or vestibular complaint; however, only $12(21.8 \%)$ of these cases underwent evaluation. Audiometric testing confirmed hearing loss in $11(91.7 \%)$ of 12 cases, with 8 exhibiting sensorineuraltype hearing loss with a downward curve compatible with ototoxicity. The grade of hearing loss ranged from mild to severe. It is worth noting in the Figure 3 that classifications of hearing loss were conducted at all frequencies (i.e., from $0.25 \mathrm{kHz}$ to $8 \mathrm{kHz}$ ).

An analysis of the time period between treatment initiation with aminoglycosides and the report of a hearing complaint revealed that both tinnitus and hearing loss were associated with duration of use (Table 1). Tinnitus was also associated with amikacin use and young age, and while hearing loss was associated with male gender. In contrast, vestibular complaints (dizziness) had the highest frequency at the beginning of treatment (Figure 3).

\section{DISCUSSION}

Although the ototoxic effects of aminoglycosides were first described in the 1940s, the underlying mechanisms remain unclear. Hearing damage associated with aminoglycoside use can include permanent hearing loss and tinnitus secondary to the degradation of sensorineural hair cells of the cochlea and/ or vestibule. Damage to cochlear hair cells is thought to be mediated by oxidative stress, starting at the base where highfrequency sounds are decoded and advancing to the apex ${ }^{6,8,13,14,15}$. In the present study, we identified hearing complaints after aminoglycoside use in $27 \%$ of medical records analyzed, similar to what has been described in the literature $(27.8 \%)^{11}$. Higher frequencies of TB and hearing complaints among male subjects in our study were also consistent with previous reports. This finding may be related to the increased tendency for ototoxicity risk factor exposure in men compared to women ${ }^{4,11,13}$.

In this study, we examined the relationship between hearing complaints and the type of aminoglycoside used, as different types of aminoglycosides affect different areas of the auditory and vestibular systems. Amikacin is described in the literature as a synthetic aminoglycoside that can cause damage to cochlear cells to produce clinical presentations including tinnitus and hearing loss. In contrast, streptomycin preferentially affects cells of the vestibule, but can also cause damage to the cells of the cochlea. In such cases, the first symptom is dizziness, followed by tinnitus and hearing loss. These mechanisms are partly consistent with our observation that amikacin and streptomycin use were associated with tinnitus. The lack of a significant association between use of these medications and hearing loss may have been due to the small number of cases included in this study. It is also noteworthy that by the time individuals perceive hearing loss, the lesion has already progressed to affect the area of the cochlea responsible for the transduction of sound in the frequencies of human speech. Therefore, some subjects may have had yet undetectable hearing loss.

Some phonemes such as [s], [f], and [t] have low intensities, such that any loss in high frequencies can hinder their perception $^{5,9}$. Difficulty hearing but not understanding what is being said can cause embarrassment and lead to social isolation, often with occupational consequences ${ }^{5}$. Because of this, it is essential to carefully consider patient complaints of hearing loss or difficulty understanding speech. A hearing aid is one possible solution in patients with damage related to aminoglycoside use; however, hearing aids are expensive, especially considering the socioeconomic level of the majority of individuals with TB in low- and middle-income countries ${ }^{16}$. The referral of patients with hearing loss to centers of medium- and high-complexity Hearing Health Programs of the Ministry of Health operated in conjunction with state governments is the most suitable option given the socioeconomic profile of the population studied.

Tinnitus was the second most frequent hearing complaint in this study. Tinnitus is defined as an auditory perception in the absence of an actual stimulus. The subjective characteristics of tinnitus complicate investigations of its pathophysiology. Tinnitus is fairly common in clinical practice; although there are no studies about its prevalence in Brazil, tinnitus affects nearly 40 million people in the United States and England ${ }^{5,9}$. Previous 


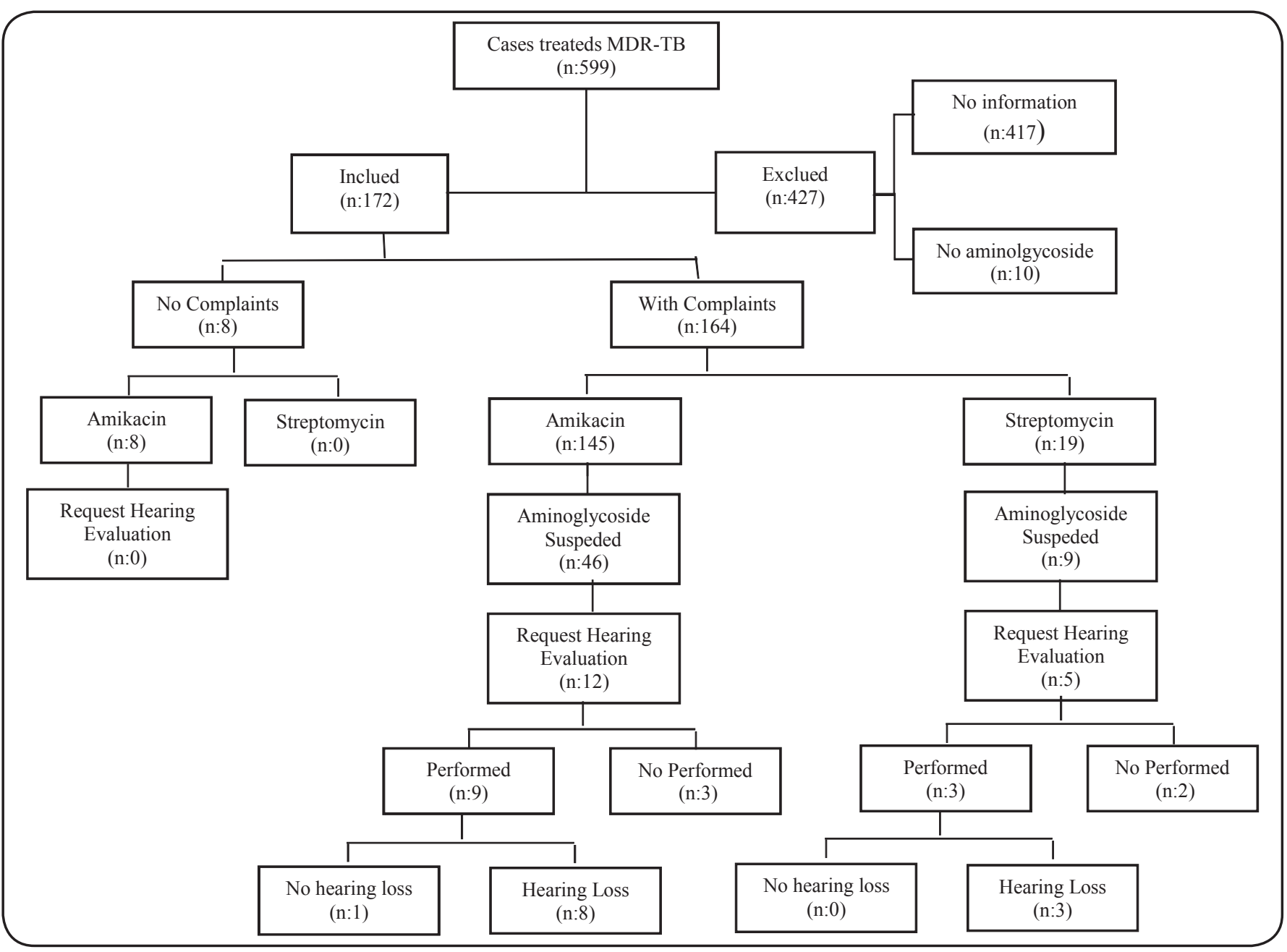

FIGURE 1 - The study on the organization chart data colletion in CRPHF (2006-2010). CRPHF: Centro de Referência Professor Helio Fraga; MDR-TB: multidrug-resistant tuberculosis.

studies have described an association between tinnitus and the use of amikacin and streptomycin as well as the appearance of tinnitus prior to hearing loss ${ }^{9}$. At present, there is no treatment for tinnitus induced by ototoxicity, as the hearing damage is irreversible. Rather, strategies to mask the perception of tinnitus can be used to prevent symptoms from interfering with day-today life. The most frequent strategies indicated are the use of background music to fall asleep, relaxation techniques, and a noise-generating hearing aid.

Dizziness is a vestibular complaint defined as an illusory sense of rotation associated with impaired perception of mobile environments 5 . Damage to the hair cells of the vestibular system (the bulb and otolith organs) can activate the reflex responses generated by head, eye, and body movements triggering dizziness $^{5,9,15}$. Although the relationship between streptomycin use and dizziness is well characterized in the literature ${ }^{5}$, we did not identify this association in our population. Nevertheless, dizziness was the most frequently reported complaint at the beginning of treatment. This can be explained by the normal physiological response to damage caused by vestibulotoxic agents; injury to the vestibular system is followed by neurological reorganization to restore corporal equilibrium

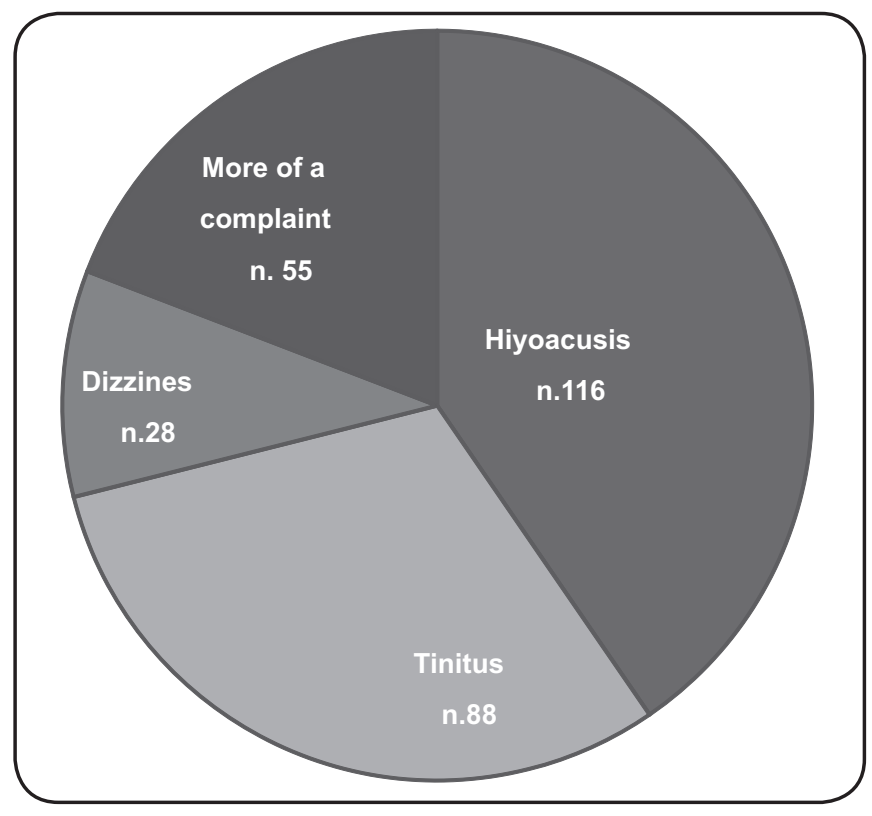

FIGURE 2 - Distribution of hearing/vestibular complaints in the studied population. 


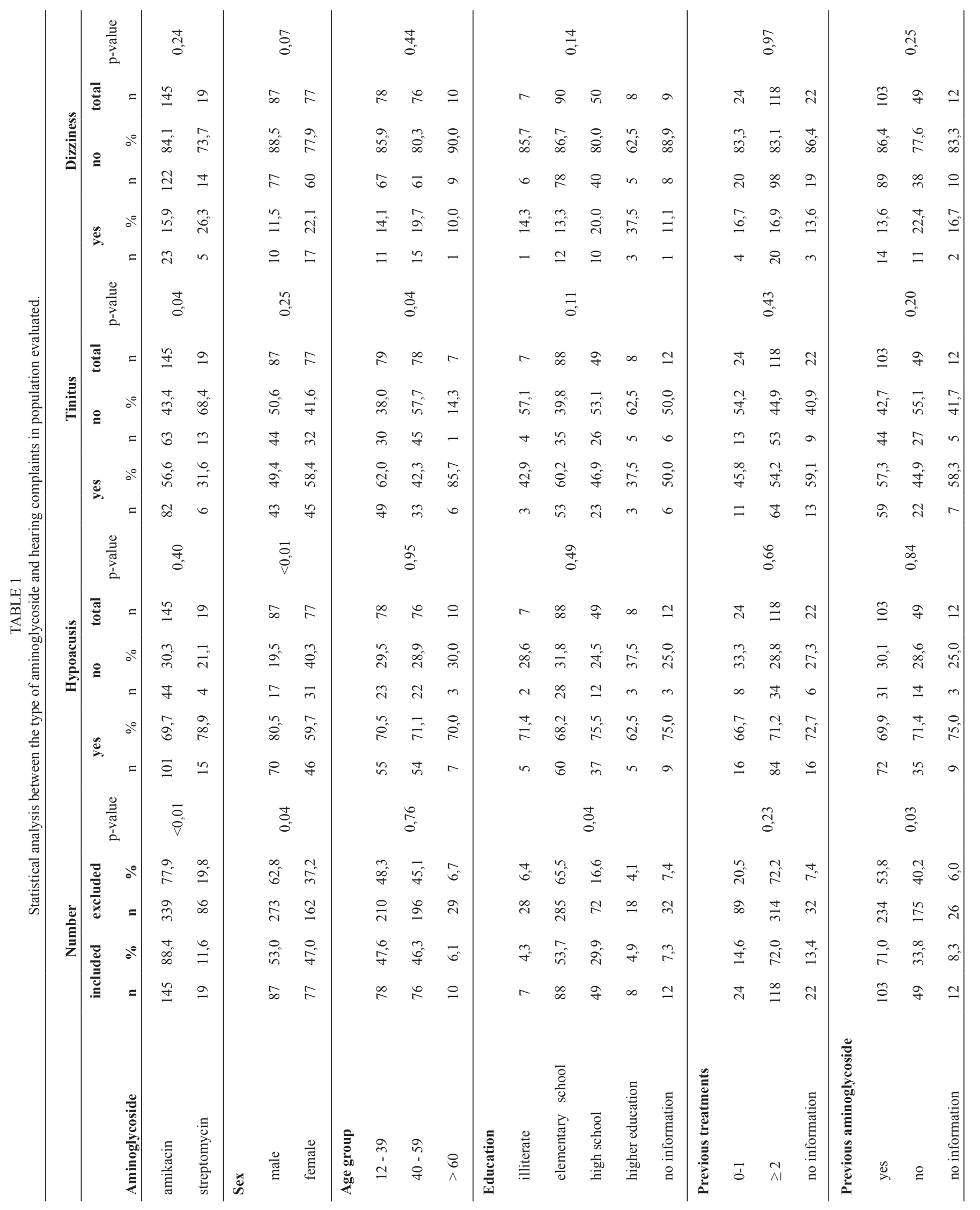




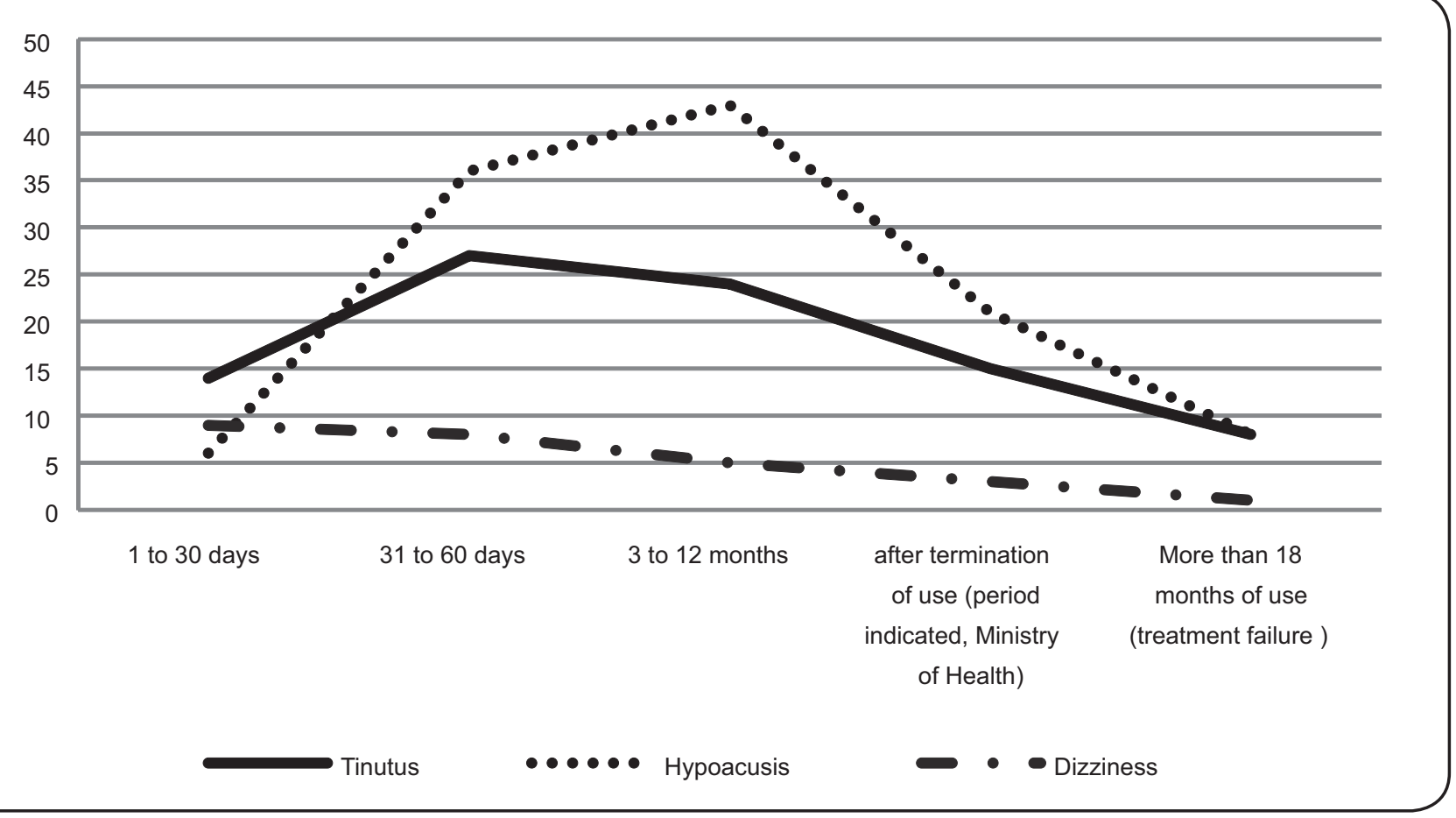

FIGURE 3 - Distribution of the relationship between the time of use and the reporting of auditory/vestibular complaints.

over time. Therefore, patients exposed to vestibulotoxic agents may initially experience some imbalance or dizziness and recover thereafter. Vestibular rehabilitation or labyrinthine rehabilitation can accelerate the process of compensation in cases of dizziness resulting from the use of ototoxic drugs.

In the present study, approximately $70 \%$ of records analyzed were excluded because there were no reported adverse events of interest. Auditory and vestibular complaints should have a prominent place in the medical records of patients with MDRTB who are treated with aminoglycosides. When a complaint was reported, physicians discontinued aminoglycoside treatment without laboratorial confirmation of hearing loss. Awareness of the consequences of a hearing loss and delays for diagnosis in the public health system likely caused physicians and patients to decide against the continuation of treatment. In our study, only $27 \%$ patients were referred for hearing assessment and of these, $91 \%$ of cases were confirmed to have hearing loss. The addition of an on-site or referral team of otolaryngologists and audiologists to TB clinics can enable the proper testing and monitoring of auditory and vestibular function in patients treated for MDR-TB.

The present study had some limitations. First, the effects or contributions of confounding variables for hearing loss such as smoking and noise exposure were not studied and are not currently known in the context of aminoglycoside ototoxicity. Our results suggest that cases of hearing loss identified in this study were due to aminoglycoside use, given the types of impairment, the period of onset, and the audiometric configuration. A high prevalence of sensorineural bilateral loss with downward curves among individuals tested suggests a possible association that should be examined in future studies. Second, the study was limited by a retrospective design and the availability of data in patient medical records/missing data. The attending medical specialists at MDR-TB reference centers do not habitually document data on auditory or vestibular complaints. Proper auditory monitoring and the careful assessment of hearing complaints should be routine procedures in treating patients with MDR-TB.

The development of standard operating protocols for monitoring hearing has not been a priority in medical practice; however, a systematic investigation into the hearing conditions of patients exposed to ototoxic drugs would: 1) support clinical decisions; 2) provide an assessment of the overall effectiveness of current clinical methods for monitoring ototoxicity; and 3) aid the development of programs for clinical application.

\section{Acknowledgments}

We thankful to the team members of the outpatient clinic at Hélio Fraga Reference Center/Fundação Oswaldo Cruz (FIOCRUZ), especially Dr. Margareth Dalcolmo and nurse Suzanne Leite.

\section{Conflicts of interest}

The authors declare that there is no conflict of interest.

\section{REFERENCES}

1. World Health Organization (WHO). Global tuberculosis Report, 2015. Control: epidemiology, strategy, financing. $20^{\text {th }}$ edition. Genève: WHO; 2015. 204p. Available from: http://apps.who.int/iris/ bitstream/10665/191102/1/9789241565059_eng.pdf?ua=1

2. Ministério da Saúde (MS). Secretaria de Vigilância em Saúde. Departamento de Vigilância das Doenças Transmissíveis. Panorama da tuberculose no Brasil: indicadores epidemiológicos e operacionais. 
Brasília: MS; 2014. 94p. Disponível em: http://bvsms.saude.gov.br/ bvs/publicacoes/panorama\%20tuberculose\%20brasil_2014.pdf

3. Schacht J, Talaska AE, Rybak LP. Cisplatin and Aminoglycoside Antibiotics: Hearing Loss and Its Prevention. Anat Rec. 2012;295(11):1837-50.

4. Dulon D, Aran JM, Zajic G, Schacht J. Comparative updake of gentamicin, netilmicin and amicacin in the guinea pig cochlea and vestibule. Antimicrob Agents Chemother. 1986;30(1):96-100.

5. Wu WJ, Sha SH, Schacht J. Recents advances in understanding aminoglycoside ototoxicity and its prevention. Audiol Neurootol. 2002;7(3): 171-4.

6. Backus RM, De Groot JCMJ, Tange RA, Huizing EH. Pathological finfings in the human auditory system following long-standing gentamicin ototoxicity. Arch Otorhinolaryngol. 1987;244(2):69-73.

7. Duggal P, Sarkar M. Audiologic monitoring of multi-drug resistant tuberculosis pacients on aminoglicoside treatment with long term follow-up. BMC Ear Nose Throat Disord. 2007;7:5. doi: $10.1186 / 1472-6815-7-5$

8. Fausti SA, Helt WJ, Phillips DS, Gordon JS, Bratt GW, Sugiura KM, et al. Early deteccion of ototoxicity using $1 / 6^{\text {th }}$-octave steps. J Am Acad Audiol. 2003;14(8):444-50

9. de Jager P, van Altena R. Hearing loss and nephrotoxicity in longterm aminoglycoside treatment in patients with tuberculosis. Int J Tuberc Lung Dis. 2002;6(7):622-7.
10. Lima MTLT, Lessa F, Aguiar-Santos AM, Medeiros Z. Alteração auditiva em pacientes com tuberculose no nordeste do Brasil. Rev Inst Med Trop. 2006;48(2):99-102.

11. Vasconcelos KA, Lima MAMT, Frota S, Ruffino Netto A, Kritski AL. Audiometric evaluation of patients treated for pulmonary tuberculosis. J Bras Pneumol. 2012;38(1):81-7.

12. Carmo LC, Silveira JAM, Marone SAM, D'Ottaviano FG, Zagati LL, von Söhsten Lins EMD. Estudo audiológico de uma população idosa brasileira. Rev Bras Otorrinolaringol. 2008;74(3):342-9.

13. Fernandez M, Morata TC. Estudo dos efeitos auditivos e extraauditivos da exposição ocupacional a ruído e vibração. Rev Bras Otorrinolaringol. 2002;68(5):705-13.

14. Kritski A. Emergência de tuberculose resistente: renovado desafio. J Bras Pneumol. 2010;36(2):157-8.

15. American Speech-Language-Hearing Association (ASHA). Guidelines for the Audiologic Management of Individuals Receiving Cochleotoxic.DrugTherapy.2002;2:81-92.Availablefrom:http://www. scielo.br/scielo.php?script $=$ sci_nlinks \&ref $=000104 \&$ pid $=$ S1808$8694200900050000400011 \& \operatorname{lng}=\mathrm{en}$

16. Melchionda V, Wyatt H, Capocci S, Garcia Medina R, Solamalai A, Katiri S, et al. Amikacin treatment for multidrug resistant tuberculosis: how much monitoring is required? Eur Respir J. 2013;42(4):1148-50. 